\title{
Evaluation of Bmp-2, VEGF, and Vitamin D Receptor Levels in the Ligamentum Flavum of Patients with Lumbar Spinal Stenosis and Disc Herniation
}

\author{
Bilal ERTUGRUL ${ }^{1}$, Bekir AKGUN ${ }^{1}$, Gokhan ARTAS ${ }^{2}$, Fatih Serhat EROL ${ }^{1}$, Fatih DEMIR ${ }^{1}$ \\ ${ }^{1}$ Firat University, School of Medicine, Department of Neurosurgery, Elazig, Turkey \\ ${ }^{2}$ Firat University, School of Medicine, Department of Clinical Pathology, Elazig, Turkey
}

Corresponding author: Bekir AKGUN bekirakgun@yahoo.com

\section{ABSTRACT}

AIM: To histopathologically evaluate and compare bone morphogenetic protein (BMP)-2, vascular endothelial growth factor (VEGF), and vitamin D receptor (VDR) levels in the ligamentum flavum (LF) of patients with lumbar spinal stenosis (LSS) and lumbar disc herniation (LDH).

MATERIAL and METHODS: Surgical specimens of the LF in 25 patients who underwent surgery for LDH and 25 patients who underwent surgery for LSS were examined histopathologically. The prevalence and severity of BMP-2, VEGF, and VDR immunoreactivity were evaluated to create histoscores (prevalence $\times$ severity), which were compared between groups.

RESULTS: The mean BMP-2 histoscore was similar in both groups. In the LSS group, the mean VEGF histoscore was significantly higher and the mean VDR histoscore was significantly lower.

CONCLUSION: Elevated VEGF and decreased VDR levels in the LF in LSS are associated with more intense inflammation and chronic process of the disease. The prominent expression of BMP-2 in the LF in both diseases suggests that BMP-2 might be affected by inflammation regardless of chronic pressure and degeneration.

KEYWORDS: Lumbar disc herniation, Spinal stenosis, BMP-2, VEGF, VDR

ABBREVIATIONS: BMP: Bone morphogenetic protein, LDH: Lumbar disc herniation, LF: Ligamentum flavum, LSS: Lumbar spinal stenosis, PLL: Posterior longitudinal ligament, VDR: Vitamin D receptor, VEGF: Vascular endothelial growth factor

\section{INTRODUCTION}

L umbar disc herniation (LDH) and lumbar spinal stenosis (LSS) are frequently encountered in neurosurgical practice. LDH can occur at any age and progresses with biochemical and histological changes in the nucleus pulposus and annulus fibrosis that result from degeneration and axial compression. Histopathological changes may also be expected in the ligamentum flavum (LF). However, LSS typically occurs in older patients and is associated with histopathological changes that include reduction of elastic fibers, fibrosis, and LF ossification $(2,5,13)$.

Bone morphogenetic proteins (BMPs) are members of the transforming growth factor-beta (TGF- $\beta$ ) family. Fifteen BMPs have been identified and divided into subgroups according to amino acid sequence similarities. BMP-2, BMP-4, and BMP-7 are active osteoinductive agents. BMP-2, which is synthesized by osteoblasts and osteocytes and primarily found in bones, is the most osteoinductive agent and plays an important role
Bilal ERTUGRUL (1) : 0000-0001-7812-3332

Bekir AKGUN (1) : 0000-0002-1244-7892

Gokhan ARTAS (1) : 0000-0001-5529-4155
Fatih Serhat EROL (D) : 0000-0001-6528-388X

Fatih DEMIR (1) : 0000-0002-6243-6884 
in ossification. In addition, it has an important role in bone differentiation and initiation of cartilage formation and may be involved in ossification of the posterior longitudinal ligament (PLL) and LF by increasing osteoblastic features of ligament cells $(6,20)$.

Vascular endothelial growth factor(VEGF) is a potent angiogenic factor that causes proliferation of vascular endothelial cells and enables vascular tube formation (11). Although it was initially defined as a mitogen specific to endothelial cells, VEGF is actually synthesized in many different cells and plays a role in several normal physiological functions, such as hematopoiesis, wound healing, and growth. VEGF also plays a role in tumor development, neovascular diseases, and several chronic inflammatory diseases (8). In inflammation, VEGF increases vascular permeability and acts as a powerful chemotacin for monocytes (4), which are active in the final stages of inflammation.

Vitamin D is a fat-soluble steroid molecule that increases calcium and phosphorus absorption in the intestines and kidneys and plays a direct or indirect role in many other physiological mechanisms. Active vitamin $D$ is involved in cellular growth and differentiation, DNA repair, apoptosis, membrane transport, adhesion, and oxidative stress and exerts its effect by binding to the vitamin D receptor (VDR) (10). This receptor has been found in tissues of the kidney, skin, prostate, breast, lung, and brain as well as macrophages of the immune system. VDR is also expressed in the cells of the nucleus pulposus and annulus fibrosus and exerts a significant effect on proteoglycan synthesis. Polymorphism of the VDR gene has been associated with disc degeneration and risk of $\operatorname{LDH}(3,21)$.

This study evaluated the levels of BMP-2, VEGF, and VDR in LF tissue specimens from patients with LDH or LSS who underwent surgery and discussed them in light of the literature data.

\section{MATERIAL and METHODS}

Following approval by the Research Ethics Committee of our university (with the date of $30^{\text {th }}$ March 2017 and the decree no. of 11).

In total, 50 patients who underwent surgery for LDH or LSS at our institution between February 2017 and January 2019 were included. All patients provided written informed consent for surgery and study participation. Patients were divided into two groups according to indication for surgery: LDH (25 patients) and LSS (25 patients). Patients with only one level LDH without apparent thickened LF and patients with only one level LSS with thickened LF were selected (Figure 1AD). Routine LF tissue specimens taken during surgery were examined histopathologically.

\section{Histopathological Examination}

LF tissue specimens were fixed with buffered $10 \%$

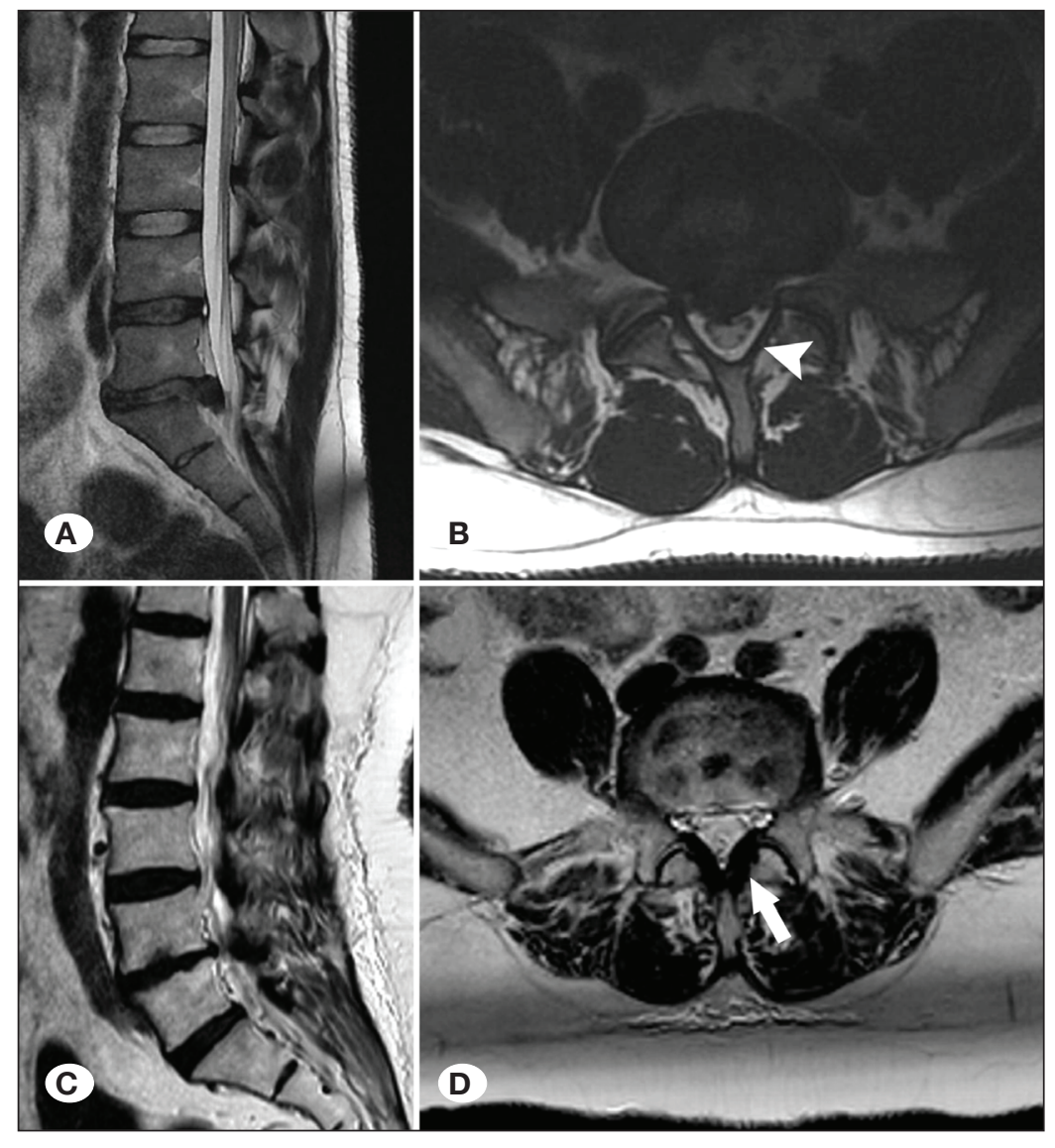

Figure 1: Sagittal (A) and axial (B) magnetic resonance imagining (MRI) images from a study patient with lumbar disc herniation (LDH; at L5-S1 level; arrow head shows not thickened ligamentum flavum [LF]). Sagittal (C) and axial (D) MRI images from a study patient with lumbar spinal stenosis (LSS; at L4-5 level; arrow shows thickened LF). 
formaldehyde solution for $48 \mathrm{~h}$, decalcified with Tris- $\mathrm{HCl}$, and then checked daily for adequate softening. Once softening was achieved, specimens were embedded in paraffin and 4- $\mu$ m-thick sections were prepared for automatic immunohistochemical staining using antibodies for BMP-2, VEGF, and VDR on polylysine slides. After staining, slides were examined and photographed using an Olympus BX51 light microscope (Figure 2A-E and 3A-E). Based on the prevalence (0.1: $<25 \%, 0.4: 26 \%-50 \%$, 0.6: $51 \%-75 \%$, and 0.9: $76 \%-$ $100 \%)$ and severity (0: none, +0.5 : very little, +1 : little, +2 : moderate, and +3 : severe) of immunoreactivity after staining, a histoscore was created (histoscore $=$ prevalence $\times$ severity).

\section{Statistical Analysis}

Statistical analyses were performed using SPSS software version 22.0 (IBM Corp., Armonk, NY, USA). Data are expressed as numbers, percentages, and means with standard deviation. The chi-square test was used to compare categorical and independent samples $t$ test was used to compare the continuous data.

\section{RESULTS}

In total, 28 patients were female (56\%) and 22 were male (44\%). Among the 25 patients in the LDH group, 10 were female (40\%) and 15 were male (60\%). In the LSS group, 18 patients were female $(72 \%)$ and 7 were male $(28 \%)$. The differences in sex and age between the groups were significant. Mean age in the LDH and LSS groups was 43.9 and 61.7 years, respectively. Patients aged $<20$ years were excluded because of possible congenital spinal stenosis.

The mean BMP-2 histoscore in LF tissue did not significantly differ between the LDH and LSS groups $(p=707)$. However, in the LSS group, the mean VEGF histoscore was significantly higher ( 0.908 vs. $0.488 ; p<0.001)$ and the mean VDR histoscore was significantly lower ( 0.128 vs. $0.208 ; p=0.040)$ than those of the LDH group (Figure 4).

\section{DISCUSSION}

Cytokines and trophic factors play a role in inflammation caused by proteoglycans that have herniated outside of the disc space as well as hypertrophy of the LF in spinal stenosis. They are also involved in ischemic and inflammatory responses resulting from pressure of neural structures $(2,22)$. Although degeneration of spinal structures cannot be avoided with current medical or surgical treatment, the mechanisms involved have not yet been elucidated. Therefore, pathophysiological and clinical studies of spinal degenerative disease are needed.

Proinflammatory cytokines released in degenerated or herniated intervertebral discs exert fibrinogenic and ossification-triggering effects on LF cells. BMP-2 plays an especially

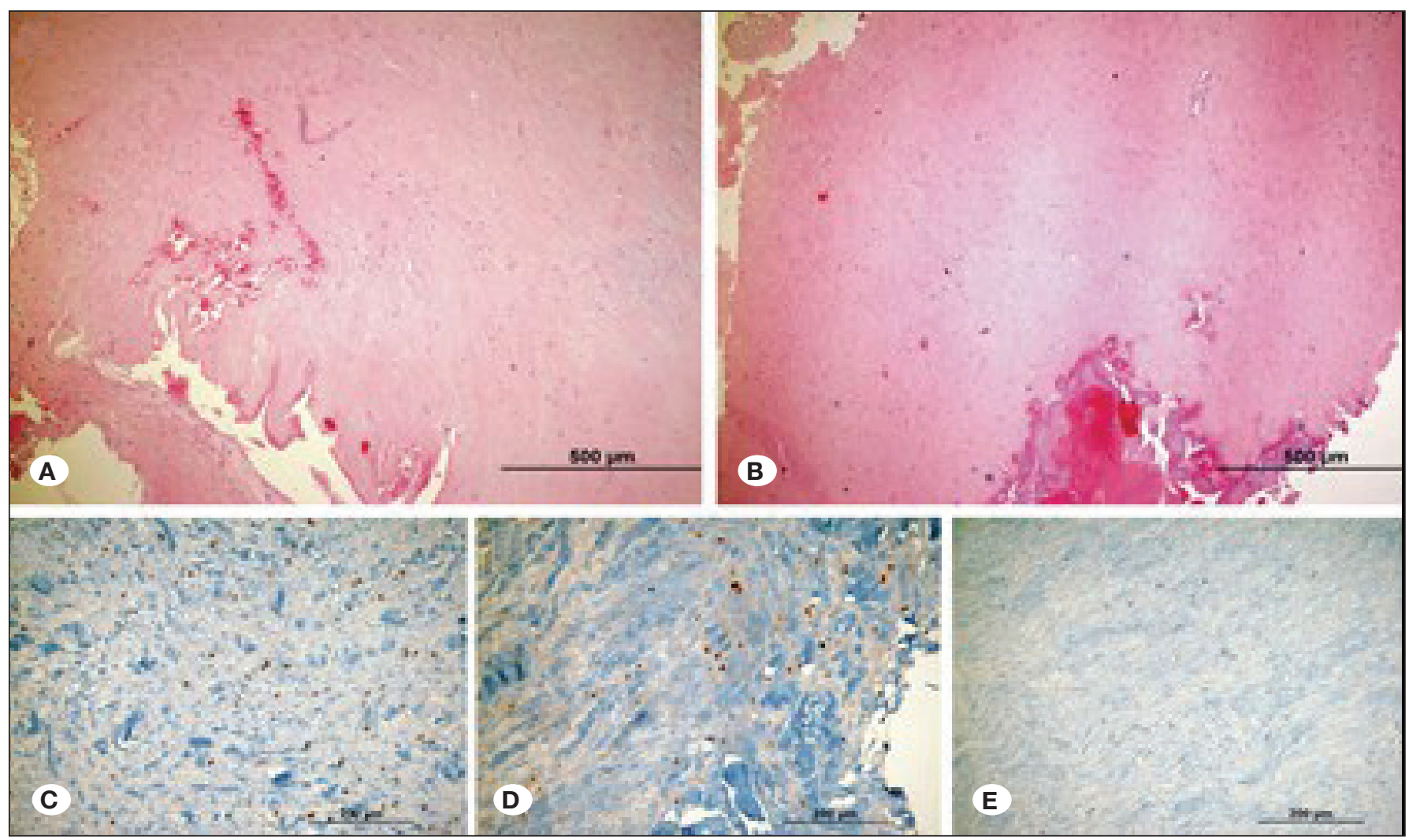

Figure 2: A, B) Microscopic view of ligamentum flavum in lumbar disk herniation (hematoxylin and eosin $\times 100$ ). C) Bone morphogenetic protein (BMP)-2 immune-reactivated view (immune peroxidase $\times 200$ ). D) Vascular endothelial growth factor (VEGF) immune-reactivated view (immune peroxidase $\times 200$ ). E) Vitamin D receptor $(V D R)$ immune-reactivated view (immune peroxidase $\times 200$ ). 

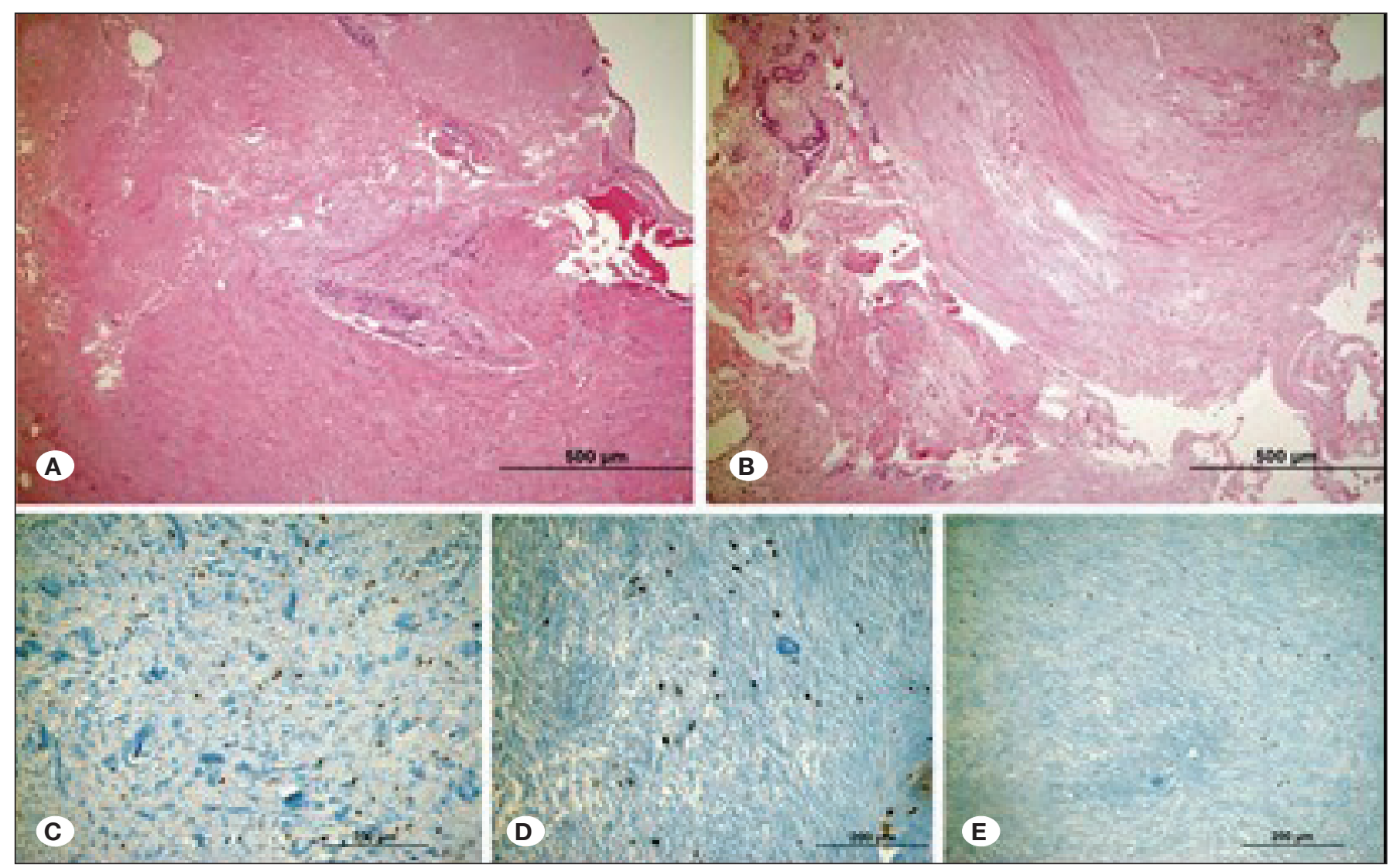

Figure 3: A, B) Microscopic view of ligamentum flavum in lumbar spinal stenosis (hematoxylin and eosin $\times 100$ ). C) Bone morphogenetic protein $(B M P)-2$ (immune peroxidase $\times 200$ ). D) Vascular endothelial growth factor (VEGF) immune-reactivated view (immune peroxidase $\times 200$ ). E) VDR immune-reactivated view (immune peroxidase $\times 200$ ).

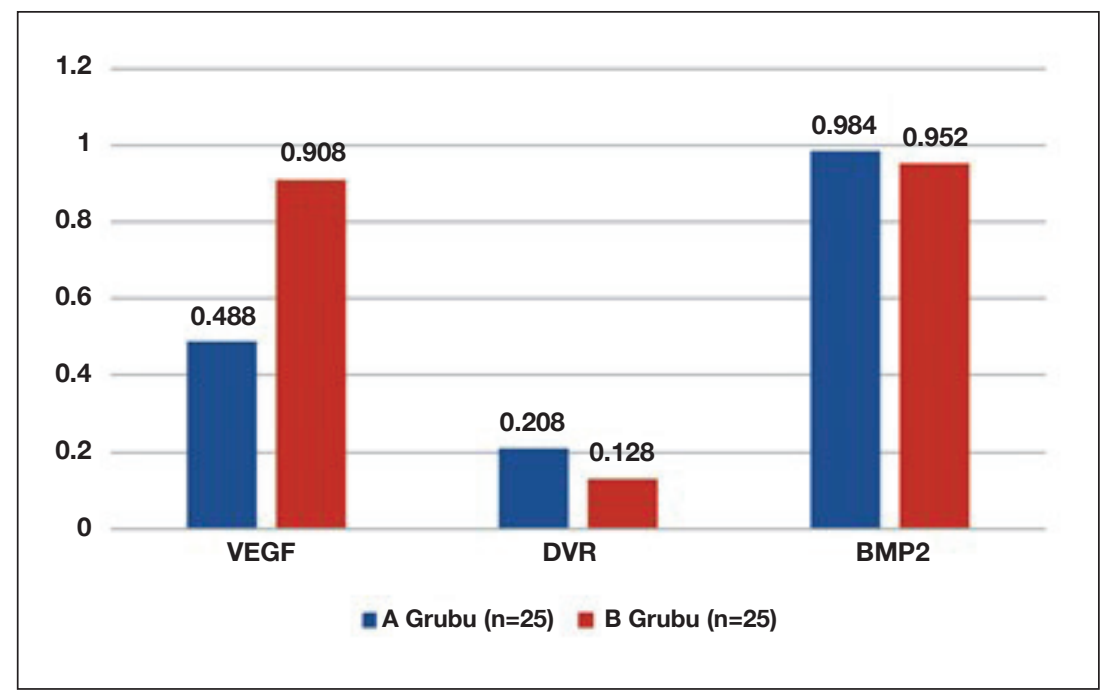

Figure 4: Comparison of histoscores for Bone morphogenetic protein (BMP)-2, Vascular endothelial growth factor (VEGF), and Vitamin $\mathrm{D}$ receptor (VDR) between the lumbar disc herniation (LDH) and lumbar spinal stenosis (LSS) groups.

important role in ossification in patients with $\operatorname{LDH}(5,18)$. Various histopathological changes, such as a decrease in elastic fibers, increase in collagen fibers, fibrosis, chondrometaplasia, and ossification, occur in LF hypertrophy associated with LSS (22). Intense BMP-2 expression has been shown in ossified LF tissue of patients with spinal stenosis (13). BMP-2 is a mem- ber of the TGF- $\beta$ super family that plays an important role in skeletal development and bone formation. It also increases osteoblast-like features of the cells in the PLL and LF $(16,20)$. BMP-2 has been used widely in spinal fusion surgery because it yields outcomes similar to the autogenous bone, enables more rapid fusion, and demonstrates high success rates 
even in revision surgery or multisegment fusions (1). In our study, significant BMP-2 expression was detected in the LF in patients with LDH and LSS, which is in line with the previous data. However, we found no significant difference in BMP-2 histoscores between these two groups. Although patients with LDH have less significant LF hypertrophy and ossification compared with those with LSS, their similar BMP-2 histoscores suggest that BMP-2 may be affected by inflammation regardless of chronic pressure and degeneration.

VEGF is a potent angiogenic factor and can be induced by agents such as interleukin-1 (IL-1) that are generated by cells in the discs. In addition, studies have shown that VEGF can be produced by several cell types, including osteoblasts (19). Haro et al. (9) speculated that VEGF may play a role in vascularization of herniated disc tissue. Furthermore, VEGF plays an important role in angiogenesis in LF hypertrophy that results from biochemical changes due to mechanical stress in patients with LSS (11). Another study reported strong and positive staining of VEGF in fibroblasts, inflammatory cells, and endothelial cells of hypertrophic LF taken from 24 patients with LSS, which was thought to represent neovascularization (12). Increased VEGF expression has also been associated with LF hypertrophy itself $(11,12)$. Lakemeier et al. (17) found that increased VEGF expression correlated with vascular density in hypertrophic LF, which is a hypovascular tissue. As angiogenesis may increase as a result of mechanical stress and inflammation, and common cytokines that are responsible for inflammation and angiogenesis also contribute to the formation of scar tissue, inflammation and angiogenesis may play a role together in LF hypertrophy. In our study, VEGF histoscores were significantly higher in the patients with LSS patients than those with LDH, which might be because LSS is more chronic and progressive and, therefore, is associated with a greater amount of local inflammation and angiogenesis.

Although vertebral disc degeneration is considered a natural process of aging, it can accelerate and cause symptoms at a younger age. Such accelerated degeneration is likely caused by genetic predisposition and environmental factors. Environmental factors, such as heavy physical activity, diabetes, excessive weight, and patient-induced factors, can trigger the disc degeneration process, exacerbating this vicious cycle $(2,13)$. Vitamin $D$ plays a role in the molecular processes involved. Remarkably, DVRs are found in osteoblasts, chondrocytes, and cells of the annulus fibrosus and nucleus pulposus (7). Although previous studies have examined VDRs in bone, vertebral disc, and glial and neural tissue, ours is the first to investigate VDR level in the LF of patients with LDH or LSS. VDR is expressed in cells of the nucleus pulposus and annulus fibrosus and has a significant effect on proteoglycan synthesis (21). VDR gene variants that modify VDR expression are involved in the pathophysiology of disc degeneration. Moreover, vitamin $\mathrm{D}$ affects the in vitro proliferation and functional regulation of nucleus pulposus and annulus fibrosis cells together via various proteins and cytokines $(2,3)$ Therefore, VDRs can be affected by inflammation. Vitamin $D$ deficiency (serum 25 -hydroxycholecalciferol $<20 \mathrm{ng} / \mathrm{ml}$ ) was highly prevalent in patients with LSS. Severe pain was associated with a higher prevalence of vitamin $D$ deficiency and osteoporosis, which could be a potential risk factor for falls and fractures (14). Ko et al. (15) reported that the prevalence of vitamin D deficiency in patients with LSS requiring decompression surgery was $76.5 \%$ in their study. Although the increase in vitamin $D$ to a certain level may be expected if the gait improves after a decompression surgery, it seems necessary to maintain 25-hydroxycholecalciferol level in serum by checking vitamin $D$ deficiency before surgery to enhance functional outcomes. Although our study detected low levels of VDR expression in the LF in both the LDH and LSS groups, the VDR histoscores were significantly lower in the LSS group, possibly because of the chronic degenerative nature of LSS or the fact that it is often detected at an older age than LDH and associated with more intense inflammation.

\section{- CONCLUSION}

Various histopathological changes may develop in the LF in LDH and LSS because of various causes. LSS develops from degenerative physiopathology over a longer period compared with LDH. In addition, stenosis of the spinal canal and/or neural foramen may cause ischemic changes that result from neural and vascular pressure. Therefore, chronic inflammation and pressure-related findings are expected. The significant increase in VEGF histoscores and the significant decrease in VDR histoscores in LSS are associated with more intense inflammation and chronic degeneration. Although BMP-2 histoscores were elevated in both LDH and LSS, they did not significantly differ. This suggests that the increase occurred because of inflammation and not LF hypertrophy or chronic pressure.

These diseases are very common. Despite several conservative and/or successful surgical interventions, satisfactory improvement is not achieved in some cases. Besides, to our knowledge, no promising therapies exist for preventing LF hypertrophy in the current literature. Therefore, identifying unknown histopathological similarities and differences between LDH and LSS may enrich the literature and facilitate the development of new treatment modalities. Further laboratory and clinical studies of BMP-2, VEGF, and VDR as potential targets for medical treatment in LDH and LSS are warranted.

\section{REFERENCES}

1. Choi SH, Koo JW, Choe D, Hur JM, Kim DH, Kang CN: Interbody fusion in degenerative lumbar spinal stenosis with additional posterolateral fusion using Escherichia coli-derived bone morphogenetic protein-2. A Pilot study. Medicine (Baltimore) 99 (24):e20477, 2020

2. Erwin WM, Fehlings MG: Intervertebral disc degeneration: Genes hold the key. World Neurosurg 80(5):131-133, 2013

3. Eser B, Cora T, Eser O, Kalkan E, Haktanir A, Erdogan MO, Solak M: Association of the polymorphisms of vitamin $D$ receptor and aggrecan genes with degenerative disc disease. Genet Test Mol Biomarkers 14(3):313-317, 2010

4. Ferrara N, Davis-Smyth T: The biology of VEGF. Endocr Rev 18:4-25, 1997 
5. Fukayama S, Nakamura T, Ikeda T, Takagi K: The effect of mechanical stress on hypertrophy of the lumbar ligamentum flavum. J Spinal Disord 8:126-130, 1995

6. Groeneveld EH, Burger EH: Bone morphogenetic proteins in human bone regeneration. Eur J Endocrinol 142:9-21, 2000

7. Gruber HE, Hoelscher G, Ingram JA, Chow Y, Loeffler B, Hanley EN: 1,25(OH)2-vitamin D3 inhibits proliferation and decreases production of monocyte chemoattractant protein-1, thrombopoietin, VEGF, and angiogenin by human annulus cells in vitro. Spine 33(7):755-765, 2008

8. Harmey JH, Dimitriadis E, Kay E, Redmond HP, BouchierHayes D: Regulation of macrophage production of vascular endothelial growth factor (VEGF) by hypoxia and transforming growth factor beta-1. Ann Surg Oncol 5:271-278, 1998

9. Haro H, Kato T, Komori H, Osada M, Shinomiya K: Vascular endothelial growth factor (VEGF)-induced angiogenesis in herniated disc resorption. J Orthop Res 20(3):409-415, 2002

10. Holick MF, Chen TC: Vitamin D deficiency: A worldwide problem with health consequences. Am J Clin Nutr 87:10801086, 2008

11. Hur JW, Kim BJ, Park JH, Kim JH, Park YK, Kwon TH, Moon $\mathrm{HJ}$ : The mechanism of ligamentum flavum hypertrophy: Introducing angiogenesis as a critical link that couples mechanical stress and hypertrophy. Neurosurgery 77(2):274281,2015

12. Jirathanathornnukul N, Limthongkul W, Yingsakmongkol W: Increased expression of vascular endothelial growth factor is associated with hypertrophic ligamentum flavum in lumbar spinal canal stenosis. J Investig Med 64(4):882-887, 2016

13. Kang YM, Suk KS, Lee BH, Kim HS, Lee KI, Park SY, Lee HM, Moon SH: Herniated intervertebral disk induces hypertrophy and ossification of ligamentum flavum. Spinal Disord Tech 27 (7):382-389, 2014

14. Kim TH, Lee BH, Lee HM, Lee SH, Park JO, Kim HS, Kim SW, Moon SH: Prevalence of vitamin D deficiency in patients with lumbar spinal stenosis and its relationship with pain. Pain Physician 16(2):165-176, 2013
15. Ko S, Chae S, Choi W, Kwon J, Choi JY: The effectiveness of vitamin $\mathrm{D}$ supplementation in functional outcome and quality of life (QoL) of lumbar spinal stenosis (LSS) requiring surgery. J Orthop Surg Res 15(1):117, 2020

16. Kon T, Yamazaki M, Tagawa M, Goto S, Terakado A, Moriya $\mathrm{H}$, Fujimura S: Bone morphogenetic protein-2 stimulates differentiation of cultured spinal ligament cells from patients with ossification of the posterior longitudinal ligament. Calcif Tissue Int 60(3):291-296, 1997

17. Lakemeier S, Schofer MD, Foltz L, Schmid R, Efe T, Rohlfs J, El-Zayat BF, Fuchs-Winkelmann S, El-Zayat BF, Paletta JRJ, Foelsch C: Expression of hypoxia-inducible factor-1a, vascular endothelial growth factor, and matrix metalloproteinases 1,3 and 9 in hypertrophied ligamentum flavum. J Spinal Disord Tech 26(7):400-681, 2013

18. Ono K, Yonenobu K, Miyamoto S, Okada K: Pathology of ossification of the posterior longitudinal ligament and ligamentum flavum. Clin Orthop Relat Res (359):18-26, 1999

19. Orlandini M, Spreafico A, Bardelli M, Rocchigiani M, Salameh A, Nucciotti S, Capperucci C, Frediani B, Oliviero S: Vascular endothelial growth factor-D activates VEGFR-3 expressed in osteoblasts inducing their differentiation. J Biol Chem 281(26):17961-17967, 2006

20. Tanaka H, Nagai E, Murata H, Tsubone T, Shirakura Y, Sugiyama T, Taguchi T, Kawai S: Involvement of bone morphogenic protein-2 (BMP-2) in the pathological ossification process of the spinal ligament. Rheumatology (Oxford) 40(10):11631168, 2001

21. Videman T, Leppävuori J, Kaprio J, Battié MC, Gibbons LE, Peltonen L, Koskenvuo M: Intragenic polymorphisms of the vitamin $D$ receptor gene associated with intervertebral disc degeneration. Spine 23(23):2477-2485, 1998

22. Yabe $Y$, Hagiwara $Y$, Ando A, Tsuchiya M, Minowa T, Takemura T. Honda M, Hatori K, Sonofuchi K, Kanazawa K, Koide M, Sekiguchi T, Itoi E: Chondrogenic and fibrotic process in the ligamentum flavum of patients with lumbar spinal canal stenosis. Spine (Phila Pa 1976) 40(7):429-435, 2015 